\title{
Events 2.0 in the transmedia branding strategy of World Cultural Heritage Sites
}

\author{
Concepción Campillo-Alhama; Alba-María Martínez-Sala
}

Nota: Este artículo se puede leer en español en:

http://www.elprofesionaldelainformacion.com/contenidos/2019/sept/campillo-martinez_es.pdf

How to cite this article:

Campillo-Alhama, Concepción; Martínez-Sala, Alba-María (2019). "Events 2.0 in the transmedia branding strategy of World Cultural Heritage Sites". El profesional de la información, v. 28, n. 5, e280509.

https://doi.org/10.3145/epi.2019.sep.09

Manuscript received on $9^{\text {th }}$ Nov 2018 Accepted on $22^{\text {th }}$ Mar 2019

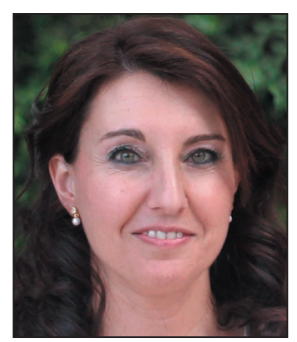

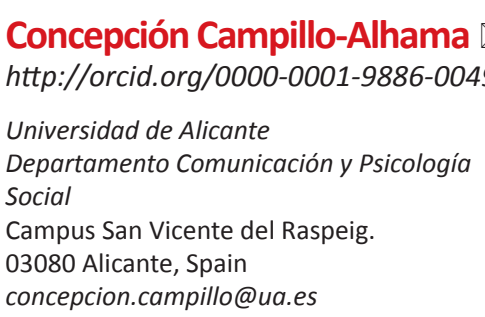

Concepción Campillo-Alhama 886-0049

Universidad de Alicante

Social

03080 Alicante, Spain

concepcion.campillo@ua.es

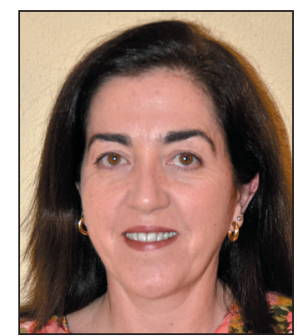

\begin{abstract}
The events designed and disseminated through social networks and from the perspective of transmedia storytelling are integrated into territorial marketing strategies as resources that reinforce the value of destinations. This type of events is key element in the construction of the World Heritage Sites (Unesco) brands. The new role of tourists as proactive users and the engagement generated towards the destination management organization are decisive elements for the online reputation management of territories. The monitoring of events let us evaluate the participation and interaction of tourist users in platforms 2.0. The aim of this research is to analyze the engagement (global, positive and negative) of events 2.0 integrated into the marketing strategy of the 40 World Cultural Heritage Sites in Spain on Facebook and Twitter. These social networks are the most frequently used in marketing strategies of destination marketing organizations. An exploratory study of events integrated in digital tourism marketing strategy of the World Cultural Heritage Sites is carried out through content analysis. Results show that there are substantial differences in the management of events 2.0. The different levels of interaction (reactions, sharing and comments) allow the destination marketing organizations (DMOs) to evaluate the rate of engagement. This latter parameter can be used as a reference for the efficient design of future events linked to the 2.0 promotion of cultural destinations.
\end{abstract}

\section{Keywords}

Tourism communication; Transmedia branding; Events 2.0; Online marketing; Social media; Social networks; Culture; World heritage; Cities; Monuments; Tourism; Archeology; History; Reputation; World Cultural Heritage Sites; Engagement; Facebook; Twitter; Unesco.

\section{Financing}

This work has been developed by the Institutional, Political and Social Advertising Research Group (Gepips) of the University of Alicante, within the framework of the I3CE Research Network Program for University Teaching of the Education Sciences Institute and the Vicerectorate of Educational Quality and Innovation of the University of Alicante (Call 2018-19). Ref.: (4338) Protocol inter-university network of collaborative work in protocol, event management and institutional relations (2010-2019). 


\section{Introduction}

The value of World Heritage Sites (WHS) is on the rise, according to reports published by Turespaña (2007), the World Tourism Organization (Unwto, 2016) and the Spanish World Heritage Cities Group (2018). This type of destinations enjoys a very significant and relevant demand worldwide.

The bet of Destination Marketing Organizations (DMO) on the strategic promotion of the WHS brand is a clear example of how it is possible to destabilize the traditional tourism demand (Troitiño-Vinuesa, 2000; Martos-Molina, 2013; Gómez-Oliva et al., 2017), which in Spain continues to be associated with sun and beach tourism (Martínez-Sala, 2018).

Tourism marketing 2.0 has transformed the destination strategies of WHS and their promotional events, whose design involves multidirectional channels and transmedia storytelling techniques.

Social media have transformed the traditional tourist into an adprosumer, who promotes and advertises the destination $(a d)$, produces information about it (pro) and consumes the tourist products and by-products (sumer) offered by the destination (Caro; Luque; Zayas, 2015).

The new role of the tourist as a proactive user during the whole process is key in the management of the reputation of destinations. The monitoring of this type of tourist allows DMO to alleviate negative comments and opinions and to manage engagement effectively as a commitment acquired with users (Huertas-Roig; Setó-Pàmies; Míguez-González, 2015; Ejarque-Bernet, 2016). This context entails a great transformation in the strategic marketing planning and the relational communication and management of the tourist destination.

In this sense, this type of events are identified as true transmedia resources that serve to reinforce the value of the destinations and consolidate their online reputation (Jiménez-Morales; De-San-Eugenio-Vela, 2009; Campillo-Alhama, 2012; Martos-Molina, 2013; Folgado-Fernández, 2014; Huertas-Roig, 2014; Campillo-Alhama; Martínez-Sala, 2017). For the WHS brand, the communication strategy of events through social networks is a challenge and a very valuable opportunity to reinforce the cultural, historical and identitary offer of destination.

This research analyzes the destination marketing strategy based on the transmedia building of the tourist brand through events 2.0, and, second, the influence of the events planned and managed by the 40 WHS in Spain through the social networks Facebook and Twitter in the generation of engagement.

\section{Destination marketing $\mathbf{2 . 0}$ and transmedia branding}

With the emergence of the Internet 1.0 and the design of static web pages in the 1990s, the face-to-face models of tourist information and service provision (e.g., visitor information center) began to coexist with their digital counterparts. It was a basically passive management model until, with the arrival of the new millennium, the Web 2.0 made it possible for tourist information to be designed not only by DMO or companies linked to the sector, but also by those tourists who visited the destination and wanted to generate their own contents and share their opinions and feelings (Xiang; Gretzel, 2010; Caro; Luque; Zayas, 2015; Huertas-Roig; Setó-Pàmies; Míguez-González, 2015; Biz et al., 2016; Chan-Olmsted; Wolter, 2017).

Tourism marketing 2.0 focuses on the inbound philosophy, which is a marketing strategy that is oriented to the consumer and potential customers rather than focused on a specific tourist product or destination. Ejarque-Bernet defines it as

"the marketing strategy that uses content, search engines and social networks to attract users" (Ejarque-Bernet, 2016, p. 455).

In the same way as it happened in other economic sectors, the communication models in the tourism sector have evolved towards transmedia branding to adapt to the features of the new tourist 2.0. This model is the result of a saturated market in which destinations need to build their own identity, brand image and personality, beyond the traditional destination information and promotion goals (Jiménez-Morales; De-San-Eugenio-Vela, 2009; Galmés-Cerezo, 2010).

The transmedia branding model makes it easier for brands to connect emotionally with consumers and generates communities of users around the lived and shared experience (Fernández-Castrillo, 2014). It consists of integrating tangible and intangible values and meanings associated with the brand's personality into a multichannel narrative that provides an integrated brand experience. The active participation of users generates engagement and builds loyalty (Tenderich, 2014; Martínez-Sala; Monserrat-Gauchi; Campillo-Alhama, 2017). The participation of users in the very development of the narrative is central to the building of the brand identity.

Under the transmedia approach, the effort of tourism brands focuses on materializing experiences through multiple channels; including events as true strategic and intentional tools (Lenderman, 2008; Campillo-Alhama, 2012) that use the narrative format to highlight the most outstanding values of the destination and generate experiences in a specific space: the destination. They are integrated in the strategies of relational communication

The transmedia branding makes it easier for brands to connect emotionally with consumers and generates communities around the lived and shared experience 
and management of the tourist destinations and their promotion is carried out, from a transmedia perspective, through social media (AIMC, 2017; IAB Spain, 2017).

Social networks are used in the planning, advertising, execution and management of events 2.0, because they provide highly efficient alternatives to promote the tourist event from a transmedia point of view and to achieve much more notoriety, engagement and influx of participants, both physical and virtual (Martos-Molina, 2013; Huertas-Roig, 2014; Fernández-Vázquez; López-Rodríguez; Arévalo-Iglesias, 2016).

\subsection{Transmedia events in tourist communication}

In the design of tourist brands the organization of transmedia events is a highly efficient resource that allows to reinforce the value of the destinations and to consolidate their brands through the relational communication and management (Martos-Molina, 2013; Huertas-Roig, 2014).

As systematically and creatively designed events, they should promote unique and singular experiences (Lenderman, 2008; Galmés-Cerezo, 2010; Pompurová et al., 2018). This creative dimension represents the point of connection between the aspects intrinsic to the narrative (storyline, rhythm, notoriety, coherence) and to the structure of the events.

They are initiatives and events of various kinds with a certain degree of significance and notoriety as they are integrated into the destination marketing strategy (Campillo-Alhama, 2012). The planning of these events involves a preliminary phase of design, an executive (or operational) phase, and evaluation phase based on ROI (return on investment) and ROE (return on engagement): both processes require systematization and evaluation mechanisms to measure the effective achievement of the objectives set by the DMO.

In the preliminary and executive phase of the event, social media can encourage the building of a network of contacts, conversation between the event's attendees and organizers, interaction between attendees and participants (backchannel) and the sharing of material (videos, images, news). After the event, the feedback of participants is evaluated and their interaction on platforms 2.0 and social networks (through reactions, shares and comments) is monitored. Facebook and Twitter are the main social networks for the promotion of these events (Event Marketing Institute; FreemanXP, 2015). As platforms where the community of users shares ideas, experiences and opinions about the event, social networks assume a crucial role in the generation of engagement. Facebook stands out as the most effective platform before and after the event, while Twitter is used the most during the development of the event, as noted in the aforementioned report.

Through social networks the community of users shares ideas, experiences and opinions about the event

In social networks, DMO and tourist users have a multidirectional communication channel to disseminate and promote destination marketing events with a transmedia orientation (Martos-Molina, 2013; Huertas-Roig, 2014).

There are also several collaborative platforms in the digital environment specifically created for event management, such as

- Tweetvite.com, that allows users to send event invitations to Twitter and Facebook contacts, receive confirmation of attendance and personalize virtual invitations; and

- Twebevent.com, that offers the possibility of following events on Twitter through hashtags, chatting between attendees, adding event information and integrating video and audio.

The efficient use of these collaborative platforms for event management, together with social media, will favor the automated management of events, their efficient promotion, greater social participation and the generation of value towards the tourist destination. And in this sense, it is pertinent to establish a well-defined preliminary strategy that integrates social and digital media with the rest of the offline communication channels that will be used in the management of events linked to the promotion and commercialization of destinations, as well as DMO's explicit desire to interact with stakeholders, betting on transparency and continuity in the conversation through platforms 2.0.

\subsection{World Heritage Sites and generation of engagement through events $\mathbf{2 . 0}$}

Cities in which sites of the Unesco World Heritage (WHS) list are located are tourist destinations whose identity is persistently linked to historical, cultural and artistic heritage. The WHS designation can be a competitive advantage over other tourist destinations (Lara-de-Vicente; López-Guzmán-Guzmán, 2004), provided integral strategies are planned to highlight the importance of such resources.

As of January 2018, Spain had a total of 40 sites designated as WHS by Unesco. This recognition boosts tourism flows and has very positive impacts in the destinations (Unesco, 2018). The marketing strategies 2.0 of WHS involve relational communication and management actions that enhance the showcase of the tourist offer (Troitiño-Vinuesa, 2000; Domínguez-Vila; Araújo-Vila, 2012); and, in this sense, the holding of tourist-cultural events as an attraction factor for visitors is undeniable. 
Table 1. World Heritage Sites (cultural heritage category), web 2.0 and social networks

\begin{tabular}{|c|c|c|c|c|c|}
\hline & \multicolumn{2}{|c|}{ Location } & \multirow{2}{*}{\begin{tabular}{|c|} 
WHS \\
Historic center of Córdoba
\end{tabular}} & \multirow{2}{*}{$\begin{array}{c}\begin{array}{c}\text { Year of } \\
\text { inscription }\end{array} \\
1984,1994\end{array}$} & \multirow{2}{*}{$\begin{array}{l}\text { DMO website and social network pages } \\
\text { http://www.turismodecordoba.org } \\
\text { https://www.facebook.com/TurismoCordobaESP } \\
\text { https://twitter.com/CordobaESP }\end{array}$} \\
\hline 1 & Municipality & Córdoba & & & \\
\hline 2 & Municipality & Granada & $\begin{array}{l}\text { Alhambra, Generalife and } \\
\text { Albayzín }\end{array}$ & 1984, 1994 & $\begin{array}{l}\text { http://www.granadatur.com } \\
\text { https://www.facebook.com/granadaturismo } \\
\text { https://twitter.com/granadaturismo }\end{array}$ \\
\hline 3 & Municipality & $\begin{array}{l}\text { Barcelona and } \\
\text { Santa Coloma de } \\
\text { Cervelló }\end{array}$ & Works of Antoni Gaudí & 1984,2005 & $\begin{array}{l}\text { http://www.barcelonaturisme.com } \\
\text { https://www.facebook.com/visitbarcelona } \\
\text { https://twitter.com/VisitBCN_ES }\end{array}$ \\
\hline 4 & Municipality & Burgos & Burgos Cathedral & 1984 & $\begin{array}{l}\text { http://www.turismoburgos.org } \\
\text { https://www.facebook.com/DestinoBurgos } \\
\text { https://twitter.com/BurgosTur }\end{array}$ \\
\hline 5 & Municipality & $\begin{array}{l}\text { San Lorenzo de El } \\
\text { Escorial (Madrid) }\end{array}$ & $\begin{array}{l}\text { Monastery and site of The } \\
\text { Escurial }\end{array}$ & 1984 & $\begin{array}{l}\text { http://www.sanlorenzoturismo.es } \\
\text { https://www.facebook.com/turismosanlorenzoescorial } \\
\text { https://twitter.com/SLorenzoTurismo }\end{array}$ \\
\hline 6 & $\begin{array}{l}\text { Autonomous } \\
\text { Community }\end{array}$ & $\begin{array}{l}\text { Asturias (municipa- } \\
\text { lities: Oviedo and } \\
\text { Lena) }\end{array}$ & $\begin{array}{l}\text { Monuments of Oviedo and } \\
\text { the Kingdom of Asturias }\end{array}$ & 1985,1998 & $\begin{array}{l}\text { https://www.turismoasturias.es } \\
\text { https://www.facebook.com/TurismoAsturias } \\
\text { https://twitter.com/TurismoAsturias }\end{array}$ \\
\hline 7 & $\begin{array}{l}\text { Autonomous } \\
\text { Community }\end{array}$ & $\begin{array}{l}\text { Cantabria, Basque } \\
\text { Country and Princi- } \\
\text { pality of Asturias }\end{array}$ & $\begin{array}{l}\text { Cave of Altamira and } \\
\text { Paleolithic cave art of } \\
\text { Northern Spain }\end{array}$ & 1985,2008 & $\begin{array}{l}\text { https://www.turismodecantabria.com } \\
\text { https://www.facebook.com/cantabriaturismo } \\
\text { https://twitter.com/cant_infinita }\end{array}$ \\
\hline 8 & Municipality & Ávila & $\begin{array}{l}\text { Old town of Ávila and its } \\
\text { extra-muros churches }\end{array}$ & 1985,2007 & $\begin{array}{l}\text { http://www.avilaturismo.com } \\
\text { https://www.facebook.com/AvilaTurismo.oficial } \\
\text { https://twitter.com/Avila_Turismo }\end{array}$ \\
\hline 9 & Municipality & Segovia & $\begin{array}{l}\text { Old town of Segovia and } \\
\text { its aqueduct }\end{array}$ & 1985 & $\begin{array}{l}\text { http://www.turismodesegovia.com } \\
\text { https://www.facebook.com/TurismodeSegovia } \\
\text { https://twitter.com/TurismoSegovia }\end{array}$ \\
\hline 10 & Municipality & $\begin{array}{l}\text { Santiago de Com- } \\
\text { postela }\end{array}$ & $\begin{array}{l}\text { Old town of Santiago de } \\
\text { Compostela }\end{array}$ & 1985 & $\begin{array}{l}\text { http://www.santiagoturismo.com } \\
\text { https://www.facebook.com/TurismodeSantiago } \\
\text { https://twitter.com/santiagoturismo }\end{array}$ \\
\hline 11 & $\begin{array}{l}\text { Autonomous } \\
\text { Community }\end{array}$ & Aragón & $\begin{array}{l}\text { Mudejar architecture of } \\
\text { Aragón }\end{array}$ & 1986, 2001 & $\begin{array}{l}\text { http://www.turismodearagon.com } \\
\text { https://www.facebook.com/TurismodeAragon } \\
\text { https://twitter.com/aragonturismo?lang=es }\end{array}$ \\
\hline 12 & Municipality & Toledo & Historic city of Toledo & 1986 & $\begin{array}{l}\text { http://www.toledo-turismo.com } \\
\text { https://www.facebook.com/toledoturismo } \\
\text { https://twitter.com/toledoturismo }\end{array}$ \\
\hline 13 & Municipality & Cáceres & Old town of Cáceres & 1986 & $\begin{array}{l}\text { http://turismo.ayto-caceres.es } \\
\text { https://www.facebook.com/turismocc } \\
\text { https://twitter.com/cde_cacerestur }\end{array}$ \\
\hline 14 & Municipality & Sevilla & $\begin{array}{l}\text { Cathedral, Alcázar and } \\
\text { Archivo de Indias in Seville }\end{array}$ & 1987 & $\begin{array}{l}\text { http://www.visitasevilla.es } \\
\text { https://www.facebook.com/Sevilla } \\
\text { https://twitter.com/sevillaciudad }\end{array}$ \\
\hline 15 & Municipality & Salamanca & Old city of Salamanca & 1988 & $\begin{array}{l}\text { http://www.salamanca.es } \\
\text { https://www.facebook.com/TurismoSalamanca } \\
\text { https://twitter.com/turisalamanca }\end{array}$ \\
\hline 16 & Municipality & $\begin{array}{l}\text { Vimbodí (county of } \\
\text { the Conca of Bar- } \\
\text { berà) (Tarragona) }\end{array}$ & Poblet monastery & 1991 & $\begin{array}{l}\text { http://www.concadebarbera.info } \\
\text { https://www.facebook.com/enoturismeconca } \\
\text { https://twitter.com/concaturisme }\end{array}$ \\
\hline 17 & $\begin{array}{l}\text { Autonomous } \\
\text { Community }\end{array}$ & $\begin{array}{l}\text { Galicia, Navarra, } \\
\text { Aragón, La Rioja } \\
\text { and Castilla y León }\end{array}$ & $\begin{array}{l}\text { Route of Santiago de } \\
\text { Compostela }\end{array}$ & 1993 & $\begin{array}{l}\text { http://www.santiagoturismo.com } \\
\text { https://www.facebook.com/CaminoXacobeo } \\
\text { https://twitter.com/camino_xacobeo }\end{array}$ \\
\hline 18 & Municipality & $\begin{array}{l}\text { Guadalupe (Cá- } \\
\text { ceres) }\end{array}$ & $\begin{array}{l}\text { Royal monastery of Santa } \\
\text { María de Guadalupe }\end{array}$ & 1993 & $\begin{array}{l}\text { http://monasterioguadalupe.com } \\
\text { https://www.facebook.com/OficinaTurismoGuadalupe } \\
\text { https://twitter.com/OTGuadalupe }\end{array}$ \\
\hline 19 & Municipality & Merida (Badajoz) & $\begin{array}{l}\text { Archaeological ensemble } \\
\text { of Mérida }\end{array}$ & 1993 & $\begin{array}{l}\text { http://www.turismomerida.org } \\
\text { https://www.facebook.com/turmerida } \\
\text { https://twitter.com/meridaciudad }\end{array}$ \\
\hline 20 & Municipality & Cuenca & $\begin{array}{l}\text { Historic walled town of } \\
\text { Cuenca }\end{array}$ & 1996 & $\begin{array}{l}\text { http://turismo.cuenca.es/ } \\
\text { https://www.facebook.com/TurismoAyuntamientodeCuenca } \\
\text { https://twitter.com/Turismo_Cuenca }\end{array}$ \\
\hline 21 & Municipality & Valencia & $\begin{array}{l}\text { Lonja de la seda of } \\
\text { Valencia }\end{array}$ & 1996 & $\begin{array}{l}\text { http://www.visitvalencia.com } \\
\text { https://www.facebook.com/turismovalencia } \\
\text { https://twitter.com/Valenciaturismo }\end{array}$ \\
\hline
\end{tabular}




\begin{tabular}{|c|c|c|c|c|c|}
\hline 22 & Province & $\begin{array}{l}\text { León (county El } \\
\text { Bierzo) }\end{array}$ & Las Médulas & 1997 & $\begin{array}{l}\text { http://turismodelbierzo.es/ } \\
\text { https://www.facebook.com/turismobierzo } \\
\text { https://twitter.com/turismobierzo }\end{array}$ \\
\hline 23 & Municipality & Barcelona & $\begin{array}{l}\text { Palau de la Música } \\
\text { Catalana and Hospital de } \\
\text { Sant Pau }\end{array}$ & 1997, 2008 & $\begin{array}{l}\text { http://www.barcelonaturisme.com } \\
\text { https://www.facebook.com/visitbarcelona } \\
\text { https://twitter.com/VisitBCN_ES }\end{array}$ \\
\hline 24 & Municipality & $\begin{array}{l}\text { San Millán de la Co- } \\
\text { golla (Valle de San } \\
\text { Millán) (La Rioja) }\end{array}$ & $\begin{array}{l}\text { San Millán Yuso and Suso } \\
\text { monasteries }\end{array}$ & 1997 & $\begin{array}{l}\text { http://www.todalarioja.com } \\
\text { https://www.facebook.com/todalariojaturismo } \\
\text { https://twitter.com/todalarioja }\end{array}$ \\
\hline 25 & Province & Salamanca & $\begin{array}{l}\text { Prehistoric rock art sites in } \\
\text { the Côa Valley and Siega } \\
\text { Verde }\end{array}$ & 1998,2010 & $\begin{array}{l}\text { http://www.salamancaemocion.es } \\
\text { https://www.facebook.com/TurismoSalamanca } \\
\text { https://twitter.com/turisalamanca }\end{array}$ \\
\hline 26 & Municipality & $\begin{array}{l}\text { Alcalá de Henares } \\
\text { (Madrid) }\end{array}$ & $\begin{array}{l}\text { University and historic pre- } \\
\text { cinct of Alcalá de Henares }\end{array}$ & 1998 & $\begin{array}{l}\text { http://www.turismoalcala.es } \\
\text { https://www.facebook.com/alcalaturismo } \\
\text { https://twitter.com/ahturismo }\end{array}$ \\
\hline 27 & $\begin{array}{l}\text { Autonomous } \\
\text { Community }\end{array}$ & $\begin{array}{l}\text { Catalonia, Andalu- } \\
\text { sia, Murcia, Valen- } \\
\text { cian Community, } \\
\text { Aragón, Castilla-La } \\
\text { Mancha }\end{array}$ & $\begin{array}{l}\text { Rock art of the Mediterra- } \\
\text { nean basin on the Iberian } \\
\text { Peninsula }\end{array}$ & 1998 & $\begin{array}{l}\text { http://www.españaescultura.es/es/monumentos/arte_ru- } \\
\text { pestre_del_arco_mediterraneo_peninsular.html } \\
\text { https://www.facebook.com/arterupestredelarcomediterraneo }\end{array}$ \\
\hline 28 & Municipality & $\begin{array}{l}\text { San Cristóbal de La } \\
\text { Laguna (Tenerife) }\end{array}$ & San Cristóbal de La Laguna & 1999 & $\begin{array}{l}\text { http://www.turismolalaguna.org } \\
\text { https://www.facebook.com/TurismoLaLaguna } \\
\text { https://twitter.com/turismo_II }\end{array}$ \\
\hline 29 & Municipality & $\begin{array}{l}\text { Atapuerca and } \\
\text { Ibeas de Juarros } \\
\text { (Burgos) }\end{array}$ & $\begin{array}{l}\text { Archaeological site of } \\
\text { Atapuerca }\end{array}$ & 2000 & $\begin{array}{l}\text { https://www.atapuerca.org } \\
\text { https://www.facebook.com/Fatapuerca } \\
\text { https://twitter.com/FATAPUERCA }\end{array}$ \\
\hline 30 & Municipality & Tarragona & $\begin{array}{l}\text { Archaeological ensemble } \\
\text { of Tárraco }\end{array}$ & 2000 & $\begin{array}{l}\text { http://www.tarragonaturisme.cat } \\
\text { https://www.facebook.com/TarragonaTurisme } \\
\text { https://twitter.com/TGNturisme }\end{array}$ \\
\hline 31 & Region & $\begin{array}{l}\text { Alta Ribagorça (Boí } \\
\text { Valley), Lleida }\end{array}$ & $\begin{array}{l}\text { Catalan Romanesque } \\
\text { churches of the Vall de Boí }\end{array}$ & 2000 & $\begin{array}{l}\text { http://www.vallboi.cat } \\
\text { https://www.facebook.com/ValldeBoi } \\
\text { https://twitter.com/ValldeBoi }\end{array}$ \\
\hline 32 & Municipality & Elche (Alicante) & Palmeral of Elche & 2000 & $\begin{array}{l}\text { http://www.visitelche.com } \\
\text { https://www.facebook.com/visitelche } \\
\text { https://twitter.com/visitelche }\end{array}$ \\
\hline 33 & Municipality & Lugo & Roman walls of Lugo & 2000 & $\begin{array}{l}\text { http://lugo.gal/es/lugo-destino-turistico-0 } \\
\text { https://www.facebook.com/LugoTurismo/about } \\
\text { https://twitter.com/LugoTurismo }\end{array}$ \\
\hline 34 & Municipality & Aranjuez (Madrid) & $\begin{array}{l}\text { Aranjuez cultural lands- } \\
\text { cape }\end{array}$ & 2001 & $\begin{array}{l}\text { https://www.aranjuez.es/turismo/patrimonio-de-la-humanidad } \\
\text { https://www.facebook.com/pages/Paisaje-cultural-de-Aranjuez } \\
\text { https://twitter.com/realessitios }\end{array}$ \\
\hline 35 & Municipality & $\begin{array}{l}\text { Úbeda and Baeza } \\
\text { (Jaén) }\end{array}$ & $\begin{array}{l}\text { Renaissance monumental } \\
\text { ensembles of Úbeda and } \\
\text { Baeza }\end{array}$ & 2003 & $\begin{array}{l}\text { http://ubedaybaezaturismo.com } \\
\text { https://www.facebook.com/ubedaybaezaturismo } \\
\text { https://twitter.com/ubedabaezaturis }\end{array}$ \\
\hline 36 & Municipality & $\begin{array}{l}\text { Portugalete and } \\
\text { Getxo }\end{array}$ & Vizcaya bridge & 2006 & $\begin{array}{l}\text { http://www.mybilbaobizkaia.eus } \\
\text { https://www.facebook.com/mybilbaoBizkaia } \\
\text { https://twitter.com/mybilbaobizkaia }\end{array}$ \\
\hline 37 & Municipality & A Coruña & Tower of Hércules & 2009 & $\begin{array}{l}\text { http://www.turismocoruna.com } \\
\text { https://www.facebook.com/turismocoruna } \\
\text { https://twitter.com/CorunaTurismo }\end{array}$ \\
\hline 38 & Island & Mallorca & $\begin{array}{l}\text { Cultural landscape of the } \\
\text { Serra de Tramuntana }\end{array}$ & 2011 & $\begin{array}{l}\text { http://www.infomallorca.net } \\
\text { https://www.facebook.com/InfoMallorca- } \\
\text { net-100824359979487 } \\
\text { https://twitter.com/InfoMallorca }\end{array}$ \\
\hline 39 & Municipality & $\begin{array}{l}\text { Almadén (Ciudad } \\
\text { Real) }\end{array}$ & $\begin{array}{l}\text { Heritage of mercury. Alma- } \\
\text { dén and Idrija }\end{array}$ & 2012 & $\begin{array}{l}\text { http://www.almaden.es/turismo.htm } \\
\text { https://www.facebook.com/pages/Patrimonio-del-mercurio }\end{array}$ \\
\hline 40 & Municipality & Antequera (Málaga) & Antequera dolmens site & 2016 & $\begin{array}{l}\text { http://turismo.antequera.es } \\
\text { https://www.facebook.com/antequera.directaatucora- } \\
\text { zon.7?fref=ts } \\
\text { https://twitter.com/ATQaTuCorazon }\end{array}$ \\
\hline
\end{tabular}

Source: Ministry of Culture and Sport of Spain (2017)

http://www.mecd.gob.es/cultura-mecd/areas-cultura/patrimonio/mc/patrimoniomundial/bienes-declarados/por-tipo-de-bien.html

Unesco (2018)

http://portal.unesco.org/es/ev.phpURL_ID=45692\&URL_DO=DO_TOPIC\&URL_SECTION=201.htmI 


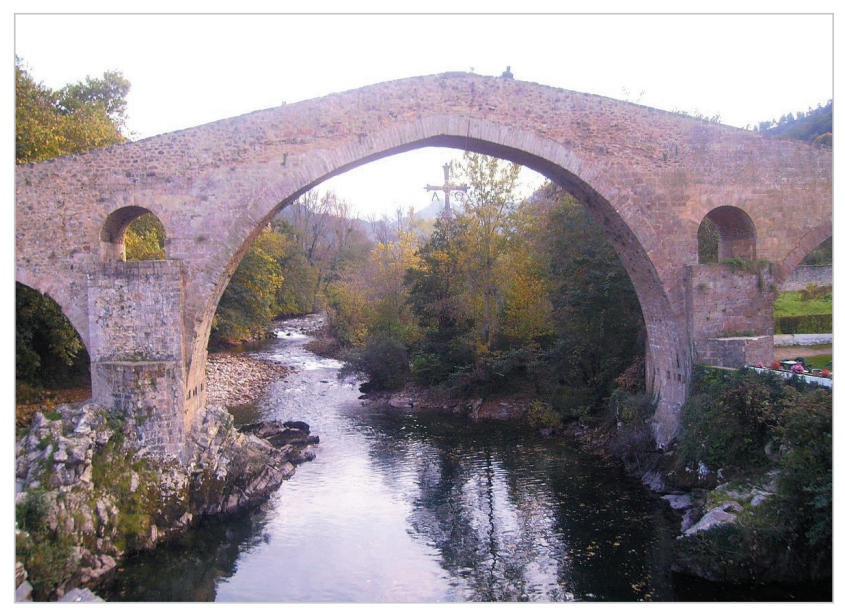

Roman bridge of Cangas de Onís, capital of the former Kingdom of Asturias https://upload.wikimedia.org/wikipedia/commons/b/b8/Puente_ Romano_de_Cangas_de_Onís.jpg

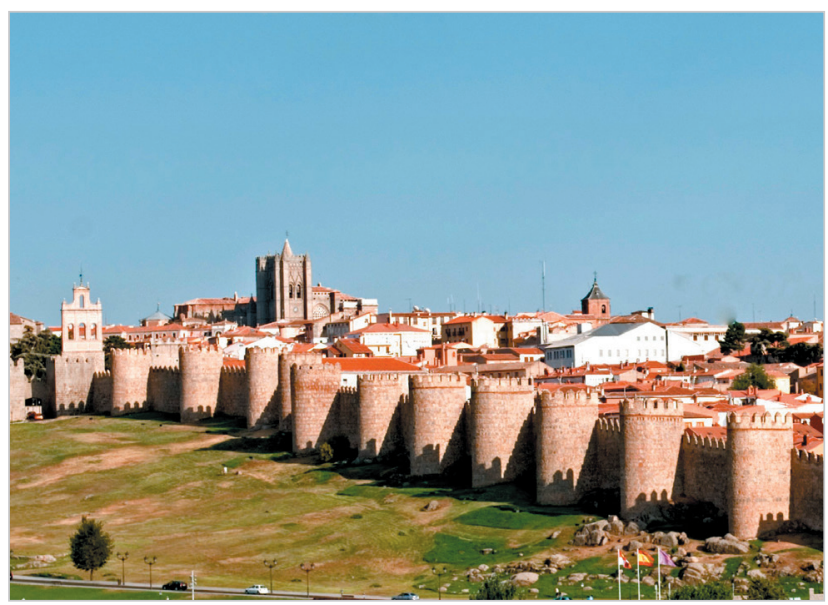

Walls of Ávila

https://commons.wikimedia.org/wiki/File:Avila_001.jpg

Numerous research studies on online engagement have been carried out to measure the effectiveness of relational communication and management 2.0: Hays, Page \& Buhalis (2013); Mendes-Thomaz, Biz \& Gândara (2013); Altamirano \& Túñez (2016); Mariani, Di-Felice \& Mura (2016); Mariné-Roig \& Huertas-Roig (2016); Harrigan et al. (2017); Mariné-Roig (2017); Pérez-Vega et al. (2018). This parameter is evaluated based on two fundamental variables: the participation and interaction of tourist users through social networks.

In the evaluation phase of the events 2.0 that promote WHS, the measurement of the ROE (return on engagement) is particularly relevant as a manifestation of the degree of cognitive and affective engagement of a user with respect to the tourist brand.

\section{Objectives and methods}

Online engagement, as a behavior of the tourist user towards the events, can be measured according to the two aforementioned variables: the degree of participation and the levels of interaction manifested on social media.

The objectives of this research are

- to analyze the engagement (global, positive and negative) of the events 2.0 linked to the marketing strategy of the 40 WHS in Spain (cultural category) based on their dissemination in the Facebook page of their DMO;

- to analyze the engagement generated through the social network Twitter.
Table 2. Variables in the formula to calculate online engagement on Facebook (global, positive and negative)

\begin{tabular}{|l|l|}
\hline \multicolumn{1}{|c|}{ Variable } & \multicolumn{1}{c|}{ Analysis items } \\
\hline Posts & Number of posts \\
\hline Reactions & $\begin{array}{l}\text { Number of reactions generated by the post: } \\
\text { Global } \\
\text { Positive: "Likes", "Love", "Haha" and "Wow". } \\
\text { Negative: "Sad" and "Angry" }\end{array}$ \\
\hline Shares & $\begin{array}{l}\text { Number of times the post has been shared } \\
\text { Comments }\end{array}$ \\
$\begin{array}{l}\text { Number of comments generated by the post: } \\
\text { Global } \\
\text { Positive } \\
\text { Negative }\end{array}$ \\
\hline
\end{tabular}

All the publications related to the events managed by the DMO linked to the municipalities, autonomous communities and regions whose monumental sties have been awarded the WHS designation by Unesco were analyzed between January 1 and December 31, 2017. The final sample of study was composed of 570 events promoted on Facebook and 505 events promoted on Twitter.

Quantitative and qualitative content analyses (Batthyány; Cabrera, 2011; González-Teruel, 2015) were performed as part of an exploratory study to measure the level of engagement (global, positive and negative) of the events 2.0 integrated into the marketing strategies of WHS.

The following formula was applied in different tourism research studies to measure engagement (Hays; Page; Buhalis, 2013; Huertas-Roig; Setó-Pàmies; Míguez-González, 2015; Mariani; Di-Felice; Mura, 2016). It is observed that it is widely accepted as a measurement tool in the academic field (Mariani; Di-Felice; Mura, 2016; Lara-Navarra et al., 2018) as well as the professional field (IAB Spain, 2017).

$$
\frac{\mathrm{n} \text { "reactions" }+\mathrm{n} \text { "shares" }+\mathrm{n} \text { "comments" }}{\text { n posts }} \times 100
$$

Based on the initial formula, below we offer two other formulas to address the qualitative-quantitative analysis of online engagement on Facebook:

- (Positive) online engagement:

$$
\frac{(\mathrm{n} \text { "Like" + "Love" + "Haha" + "Wow") + n "Share" + n positive comments }}{\text { n posts }} \times 100
$$


- (Negative) online engagement:

$\frac{(\mathrm{n} \text { "Sad" + "Angry") }+\mathrm{n} \text { negative comments }}{\mathrm{n} \text { posts }} \times 100$

In the case of Twitter, the following formulas are used to carry out a qualitative-quantitative analysis of online engagement:

- (Positive) online engagement:

$\frac{\text { n "Like" + n "retweet" + n positive comments }}{\text { n tweets }} \times 100$

$$
\mathrm{n} \text { tweets }
$$

- (Negative) online engagement:

$$
\frac{\mathrm{n} \text { negative comments }}{\mathrm{n} \text { tweets }} \times 100
$$

\section{Results}

This section presents the results of the application of the formulas established to measure online engagement (global, positive and negative) generated by DMO among tourist users through the events 2.0 that constitute our object of study. Table 4 presents the results corresponding to the online engagement generated on Facebook.

\begin{tabular}{|c|c|c|c|c|c|c|c|c|c|}
\hline & World Heritage Site & Events & Posts & Reactions & Shares & $\begin{array}{c}\text { Com- } \\
\text { ments } \\
(+-)\end{array}$ & $\begin{array}{l}\text { Global } \\
\text { enga- } \\
\text { gement }\end{array}$ & Positive & Negative \\
\hline 1 & Historic center of Córdoba & 11 & 16 & 2,418 & 2,566 & $62+0-$ & 5,372 & 5,372 & 0 \\
\hline 2 & Alhambra, Generalife and Albaicín, Granada & 17 & 24 & 2,810 & 771 & $36+2-$ & 3,739 & 3,731 & 8 \\
\hline 3 & Works of Antoni Gaudí & 10 & 13 & 11,002 & 626 & $288+2-$ & 13,859 & 13,841 & 17 \\
\hline 4 & Burgos cathedral & 2 & 2 & 136 & 64 & $3+0-$ & 350 & 350 & 0 \\
\hline 5 & Monastery and site of The Escurial & 54 & 155 & 426 & 92 & $3+0-$ & 520 & 520 & 0 \\
\hline 6 & Monuments of Oviedo and the Kingdom of Asturias & 5 & 5 & 9,459 & 2,338 & $235+2-$ & 16,537 & 16,497 & 40 \\
\hline 7 & $\begin{array}{l}\text { Cave of Altamira and Paleolithic cave art of Nor- } \\
\text { thern Spain }\end{array}$ & 8 & 10 & 976 & 2,278 & $144+3-$ & 4,724 & 4,693 & 31 \\
\hline 8 & Old town of Ávila and its extra-muros churches & 19 & 113 & 5,979 & 1,999 & $65+5-$ & 8,040 & 8,029 & 11 \\
\hline 9 & Old town of Segovia and its aqueduct & 40 & 99 & 2,131 & 1,808 & $43+5-$ & 3,987 & 3,982 & 5 \\
\hline 10 & Old town of Santiago de Compostela & 14 & 28 & 2,261 & 427 & $29+4-$ & 2,806 & 2,790 & 16 \\
\hline 11 & Mudéjar architecture of Aragón & 5 & 10 & 285 & 74 & $3+0-$ & 389 & 389 & 0 \\
\hline 12 & Historic city of Toledo & 14 & 25 & 2,626 & 1,035 & $49+1-$ & 3,861 & 3,857 & 4 \\
\hline 13 & Old town of Cáceres & 28 & 65 & 1,302 & 877 & $46+1-$ & 2,251 & 2,248 & 4 \\
\hline 14 & Cathedral, Alcázar and Archivo de Indias in Seville & 6 & 10 & 3,965 & 963 & $75+3-$ & 5,708 & 5,678 & 30 \\
\hline 15 & Old city of Salamanca & 33 & 77 & 3,011 & 946 & $38+1-$ & 4,008 & 4,006 & 1 \\
\hline 16 & Poblet monastery & 13 & 15 & 153 & 14 & $2+0-$ & 180 & 180 & 0 \\
\hline 17 & Route of Santiago de Compostela & 30 & 88 & 5,521 & 178 & $85+18-$ & 5,816 & 5,787 & 29 \\
\hline 18 & Royal monastery of Santa María de Guadalupe & 3 & 4 & 54 & 0 & 0 & 54 & 54 & 0 \\
\hline 19 & Archaeological ensemble of Mérida & 69 & 242 & 3,561 & 2,466 & $55+4-$ & 6,051 & 6,050 & 2 \\
\hline 20 & Historic walled town of Cuenca & 13 & 18 & 202 & 42 & $3+1-$ & 266 & 261 & 6 \\
\hline 21 & Lonja de la seda de Valencia & 3 & 3 & 500 & 313 & $8+1-$ & 1,113 & 1,080 & 33 \\
\hline 22 & Las Médulas & 5 & 7 & 477 & 171 & $1+0$ & 662 & 662 & 0 \\
\hline 23 & Palau de la Música Catalana and Hospital de Sant Pau & 1 & 1 & 624 & 48 & $14+0-$ & 2,072 & 2,072 & 0 \\
\hline 24 & San Millán Yuso and Suso monasteries & - & - & - & - & - & 0 & 0 & 0 \\
\hline 25 & $\begin{array}{l}\text { Prehistoric rock art sites in the Côa Valley and Siega } \\
\text { Verde }\end{array}$ & - & - & - & - & - & 0 & 0 & 0 \\
\hline 26 & $\begin{array}{l}\text { University and historic precinct of Alcalá de } \\
\text { Henares }\end{array}$ & - & - & - & - & - & 0 & 0 & 0 \\
\hline 27 & $\begin{array}{l}\text { Rock art of the Mediterranean basin of the Iberian } \\
\text { Peninsula }\end{array}$ & - & - & - & - & - & 0 & 0 & 0 \\
\hline 28 & San Cristóbal de La Laguna & - & - & - & - & - & 0 & 0 & 0 \\
\hline
\end{tabular}

Table 4. Interaction and engagement generated by events 2.0 linked to WHS on Facebook

\begin{tabular}{|c|c|}
\hline Variable & Analysis items \\
\hline Tweets & Number of tweets \\
\hline Reactions & $\begin{array}{l}\text { Number of reactions generated by the tweet: } \\
\text { Total reactions: They are considered positive } \\
\text { No reactions: They are considered negative }\end{array}$ \\
\hline Shares & Number of times the tweet has been shared (retweet) \\
\hline Comments & $\begin{array}{l}\text { Number of comments generated by the tweet: } \\
\text { Global } \\
\text { Positive } \\
\text { Negative }\end{array}$ \\
\hline
\end{tabular}

(global, positive and negative) 


\begin{tabular}{|c|c|c|c|c|c|c|c|c|c|}
\hline 29 & Archaeological site of Atapuerca & - & - & - & - & - & $\mathbf{0}$ & 0 & 0 \\
\hline 30 & Archaeological ensemble of Tárraco & 34 & 78 & 487 & 126 & $9+6-$ & 632 & 625 & 8 \\
\hline 31 & Catalan Romanesque churches of Vall de Boí & 31 & 101 & 3,457 & 473 & $45+2-$ & 3,977 & 3,973 & 4 \\
\hline 32 & Palmeral of Elche & 15 & 39 & 243 & 909 & $56+3-$ & 1,303 & 1,294 & 10 \\
\hline 33 & Roman walls of Lugo & 12 & 78 & 2,107 & 1,216 & $39+7-$ & 3,382 & 3,373 & 9 \\
\hline 34 & Aranjuez cultural landscape & - & - & - & - & - & 0 & 0 & 0 \\
\hline 35 & $\begin{array}{l}\text { Renaissance monumental ensembles of Úbeda } \\
\text { and Baeza }\end{array}$ & 20 & 54 & 667 & 176 & $17+10-$ & 893 & 873 & 20 \\
\hline 36 & Vizcaya bridge & 13 & 44 & 64 & 20 & $2+0-$ & 89 & 89 & 0 \\
\hline 37 & Tower of Hércules & 12 & 12 & 4,433 & 514 & $72+5-$ & 5,589 & 5,547 & 42 \\
\hline 38 & Cultural landscape of the Serra de Tramuntana & 16 & 36 & 7 & 0 & 0 & 7 & 7 & 0 \\
\hline 39 & Heritage of mercury. Almadén and Idrija & 3 & 5 & 0 & 0 & 0 & 0 & 0 & 0 \\
\hline 40 & Antequera dolmens site & 19 & 31 & 1,814 & 656 & $39+4-$ & 2,612 & 2,596 & 16 \\
\hline
\end{tabular}

Graph 1 shows the global engagement generated through the social network Facebook. The representation of this parameter allows us to compare the degree of engagement of tourist users after exposure and interaction with events 2.0 linked to the 40 WHS in Spain, in the selected period.

The engagement acquired by tourist users is outstanding in the case of three WHS:

- Monuments of Oviedo and the Kingdom of Asturias (value = 16,537);

- Works of Antoni Gaudí (value $=13,859$ ); and

- Old town of Ávila and its extra-muros churches (value $=8,040$ ).

In contrast, there are six WHS that do not operate any event as part of their destination marketing strategies:

- San Millán Yuso and Suso monasteries;

- Prehistoric rock art sites in the Côa Valley and Siega Verde;

- University and historic precinct of Alcalá de Henares;

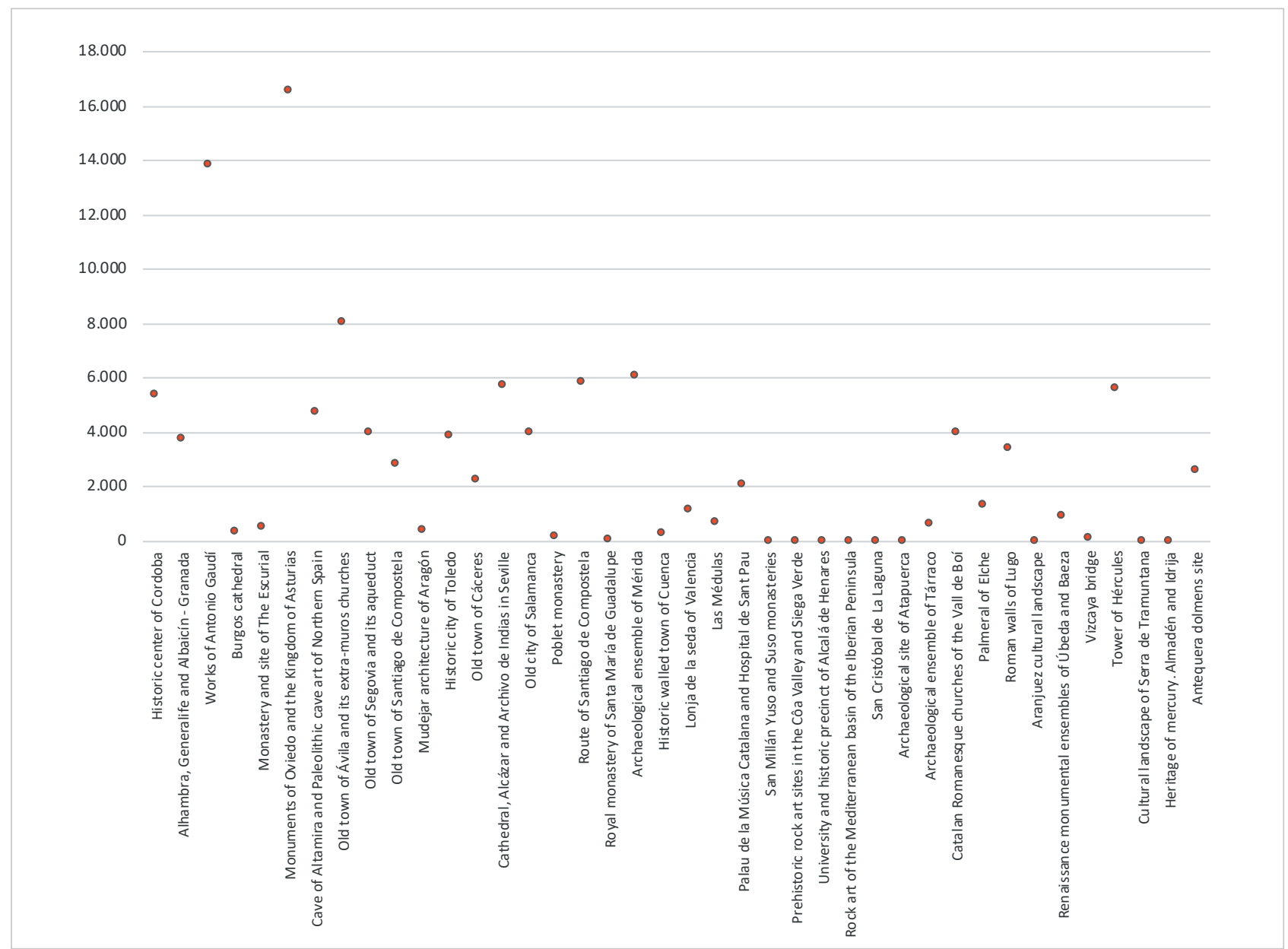

Graph 1. Global engagement on Facebook. The sites are ordered chronologically by the date they were declared World Cultural Heritage, as stated by the Spanish Ministry of Culture and Sport. 


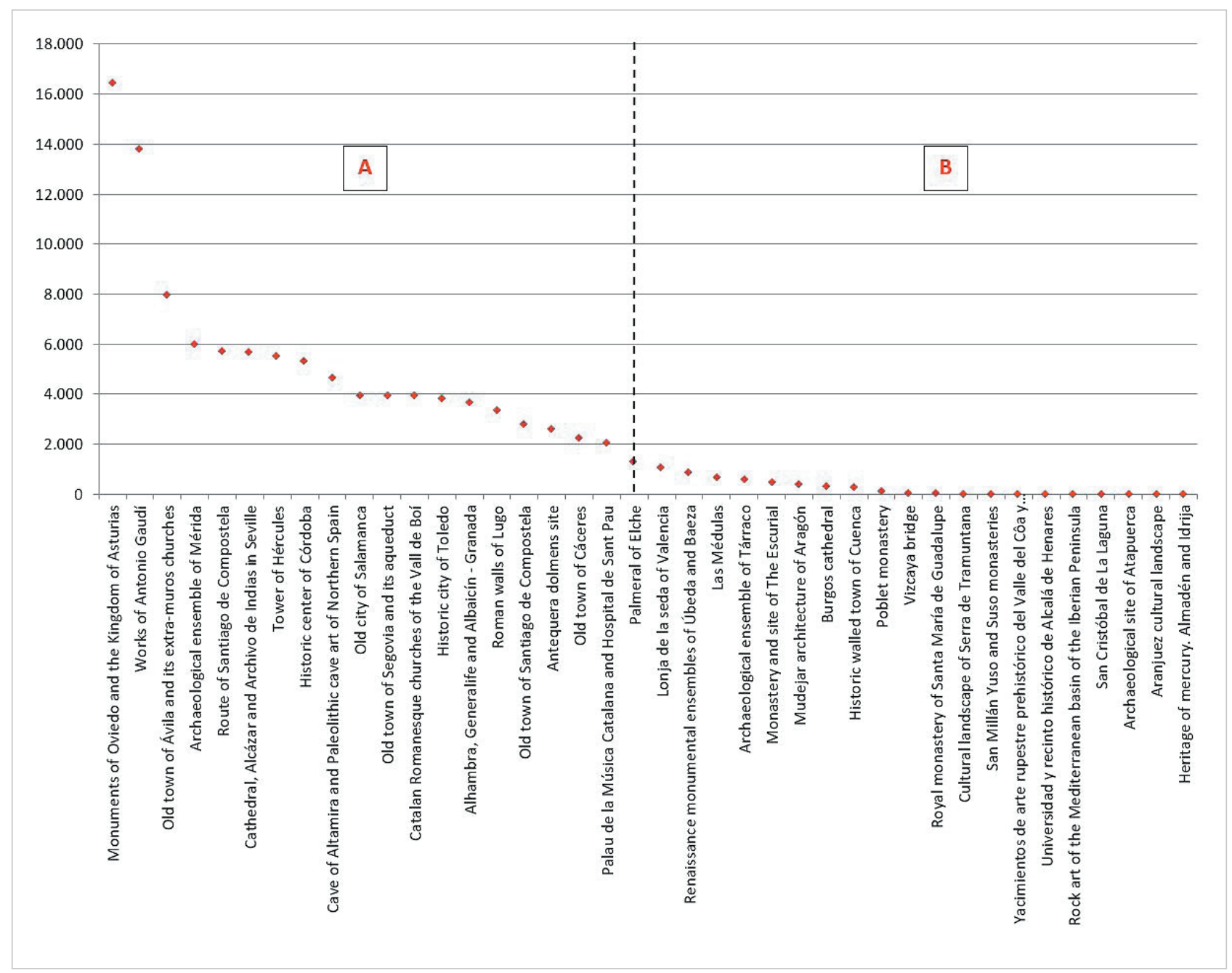

Graph 2. Positive engagement on Facebook

- Rock art of the Mediterranean basin on the Iberian Peninsula;

- San Cristóbal de La Laguna; and

- Archaeological site of Atapuerca.

We also found a WHS (Heritage of mercury. Almadén and Idrija) that has organized some events but did not generate any interaction or engagement on social network users.

Given the qualitative research results obtained on engagement, which are shown in Table 4, Graph 2 represents only the positive engagement index on Facebook obtained by each World Heritage Sites. As the negative engagement is practicaIly nil (the negative reactions are limited to $0.03 \%$ and the percentage of negative comments on the total is only $5.5 \%$ ), we consider it as a residual research result. Two levels of commitment are established through the median as the central value in a series of data that segments two differentiated areas.

The segmentation of two groups (A and B) in Graph 2 highlights the WHS that have generated the highest level of positive engagement through interaction with events 2.0. As we can see:

- Group A contains the WHS with the highest index of global engagement (Graph 1);

- In this downward progression of values, group B includes the WHS with the lowest efficiency index in the generation of engagement with tourist users.

Table 5 reflects the interaction and engagement generated in tourist users through events 2.0 on Twitter accounts of the 40 WHS.

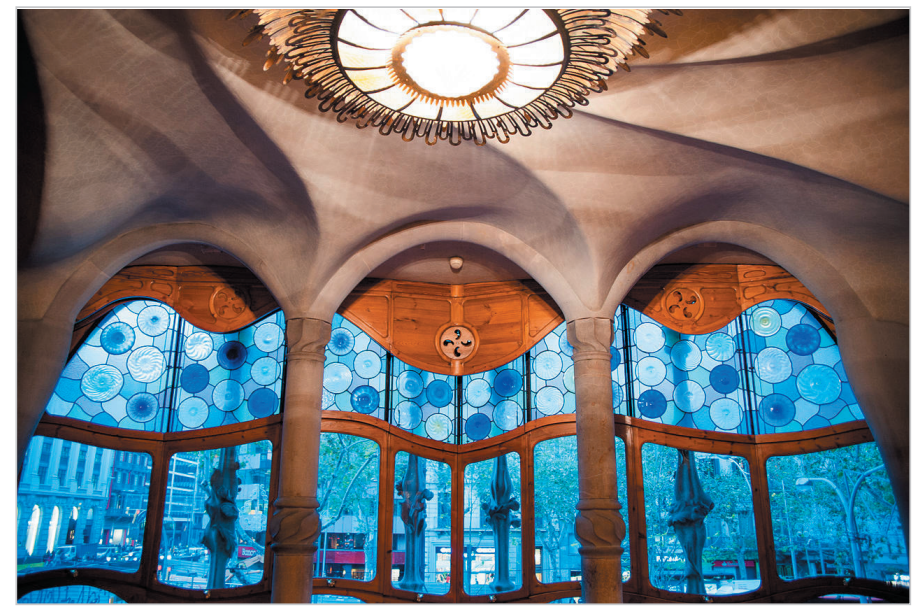

Work of Antoni Gaudí. Casa Batlló, Barcelona. https://en.wikipedia.org/wiki/Casa_Batlló 
Table 5. Interaction and engagement with events 2.0 linked to WHS on Twitter

\begin{tabular}{|c|c|c|c|c|c|c|c|c|c|}
\hline & World Heritage Site & Events & Tweets & Reactions & Shares & $\begin{array}{l}\text { Comments } \\
(+,-)\end{array}$ & $\begin{array}{l}\text { Global en- } \\
\text { gagement }\end{array}$ & Positive & Negative \\
\hline 1 & Historic center of Córdoba & 7 & 10 & 149 & 229 & 0 & 378 & 378 & 0 \\
\hline 2 & $\begin{array}{l}\text { Alhambra, Generalife and Albaicín, } \\
\text { Granada }\end{array}$ & 17 & 29 & 268 & 234 & $4+0$ & 516 & 516 & 0 \\
\hline 3 & Works of Antoni Gaudí & 12 & 20 & 55 & 38 & $2+0-$ & 103 & 103 & 0 \\
\hline 4 & Burgos cathedral & 1 & 20 & 56 & 52 & $1+0$ & 113 & 113 & 0 \\
\hline 5 & Monastery and site of The Escurial & 21 & 61 & 1 & 4 & 0 & 5 & 5 & 0 \\
\hline 6 & $\begin{array}{l}\text { Monuments of Oviedo and the King- } \\
\text { dom of Asturias }\end{array}$ & 9 & 20 & 740 & 558 & $15+0-$ & 1,373 & 1,373 & 0 \\
\hline 7 & $\begin{array}{l}\text { Cave of Altamira and Paleolithic cave } \\
\text { art of Northern Spain }\end{array}$ & 10 & 32 & 587 & 448 & $10+1-$ & 1,069 & 1,066 & 3 \\
\hline 8 & $\begin{array}{l}\text { Old town of Ávila and its extra-muros } \\
\text { churches }\end{array}$ & 26 & 254 & 1285 & 1343 & $28+6-$ & 2,641 & 2,639 & 2 \\
\hline 9 & Old town of Segovia and its aqueduct & 54 & 138 & 397 & 361 & $10+2-$ & 767 & 765 & 1 \\
\hline 10 & Old town of Santiago de Compostela & 31 & 90 & 181 & 113 & $4+1-$ & 300 & 298 & 1 \\
\hline 11 & Mudejar Architecture of Aragón & 14 & 53 & 352 & 235 & $5+0-$ & 596 & 596 & 0 \\
\hline 12 & Historic city of Toledo & 18 & 43 & 148 & 139 & $4+0-$ & 296 & 296 & 0 \\
\hline 13 & Old town of Cáceres & - & - & - & - & - & 0 & 0 & 0 \\
\hline 14 & $\begin{array}{l}\text { Cathedral, Alcázar and Archivo de } \\
\text { Indias in Seville }\end{array}$ & 11 & 34 & 599 & 465 & $4+0-$ & 1,076 & 1,076 & 0 \\
\hline 15 & Old city of Salamanca & - & - & - & - & - & $\mathbf{0}$ & 0 & 0 \\
\hline 16 & Poblet monastery & 1 & 1 & 1 & 3 & 0 & 4 & 4 & 0 \\
\hline 17 & Route of Santiago de Compostela & 28 & 83 & 524 & 301 & $1+0-$ & 826 & 826 & 0 \\
\hline 18 & $\begin{array}{l}\text { Royal monastery of Santa María de } \\
\text { Guadalupe }\end{array}$ & 4 & 7 & 1 & 0 & 0 & 1 & 1 & 0 \\
\hline 19 & Archaeological ensemble of Mérida & - & - & - & - & - & 0 & 0 & 0 \\
\hline 20 & Historic walled town of Cuenca & - & - & - & - & - & 0 & 0 & 0 \\
\hline 21 & Lonja de la seda de Valencia & 5 & 10 & 138 & 113 & $4+0-$ & 291 & 291 & 0 \\
\hline 22 & Las Médulas & 4 & 10 & 13 & 18 & 0 & 31 & 31 & 0 \\
\hline 23 & $\begin{array}{l}\text { Palau de la Música Catalana and Hospi- } \\
\text { tal de Sant Pau }\end{array}$ & 2 & 12 & 15 & 16 & 0 & 31 & 31 & 0 \\
\hline 24 & San Millán Yuso and Suso monasteries & - & - & - & - & - & $\mathbf{0}$ & 0 & 0 \\
\hline 25 & $\begin{array}{l}\text { Prehistoric rock art sites in the Côa } \\
\text { Valley and Siega Verde }\end{array}$ & - & - & - & - & - & 0 & 0 & 0 \\
\hline 26 & $\begin{array}{l}\text { University and historic precinct of } \\
\text { Alcalá de Henares }\end{array}$ & 24 & 131 & 401 & 477 & $7+1-$ & 884 & 883 & 1 \\
\hline 27 & $\begin{array}{l}\text { Rock art of the Mediterranean basin on } \\
\text { the Iberian Peninsula }\end{array}$ & - & - & - & - & - & $\mathbf{0}$ & 0 & 0 \\
\hline 28 & San Cristóbal de La Laguna & 36 & 211 & 266 & 171 & $4+1-$ & 439 & 439 & 0 \\
\hline 29 & Archaeological site of Atapuerca & - & - & - & - & - & 0 & 0 & 0 \\
\hline 30 & Archaeological ensemble of Tárraco & 34 & 389 & 656 & 554 & $14+1-$ & 1,214 & 1,214 & 0 \\
\hline 31 & $\begin{array}{l}\text { Catalan Romanesque churches of Vall } \\
\text { de Boí }\end{array}$ & 19 & 95 & 662 & 240 & $8+0-$ & 910 & 910 & 0 \\
\hline 32 & Palmeral of Elche & 15 & 89 & 396 & 355 & $5+1-$ & 758 & 757 & 1 \\
\hline 33 & Roman walls of Lugo & 20 & 75 & 178 & 182 & $0+2-$ & 363 & 360 & 3 \\
\hline 34 & Aranjuez cultural landscape & 26 & 178 & - & - & - & 0 & 0 & 0 \\
\hline 35 & $\begin{array}{l}\text { Renaissance monumental ensembles of } \\
\text { Úbeda and Baeza }\end{array}$ & - & - & - & - & - & 0 & 0 & 0 \\
\hline 36 & Vizcaya bridge & 13 & 14 & 25 & 26 & 0 & 51 & 51 & 0 \\
\hline 37 & Tower of Hércules & 20 & 81 & 413 & 292 & $6+1-$ & 714 & 712 & 1 \\
\hline 38 & $\begin{array}{l}\text { Cultural landscape of the Serra de } \\
\text { Tramuntana }\end{array}$ & 3 & 6 & 0 & 0 & 0 & 0 & 0 & 0 \\
\hline 39 & Heritage of mercury. Almadén and Idrija & - & - & - & - & - & 0 & 0 & 0 \\
\hline 40 & Antequera dolmens site & 23 & 89 & 277 & 350 & $9+3-$ & 640 & 637 & 3 \\
\hline
\end{tabular}




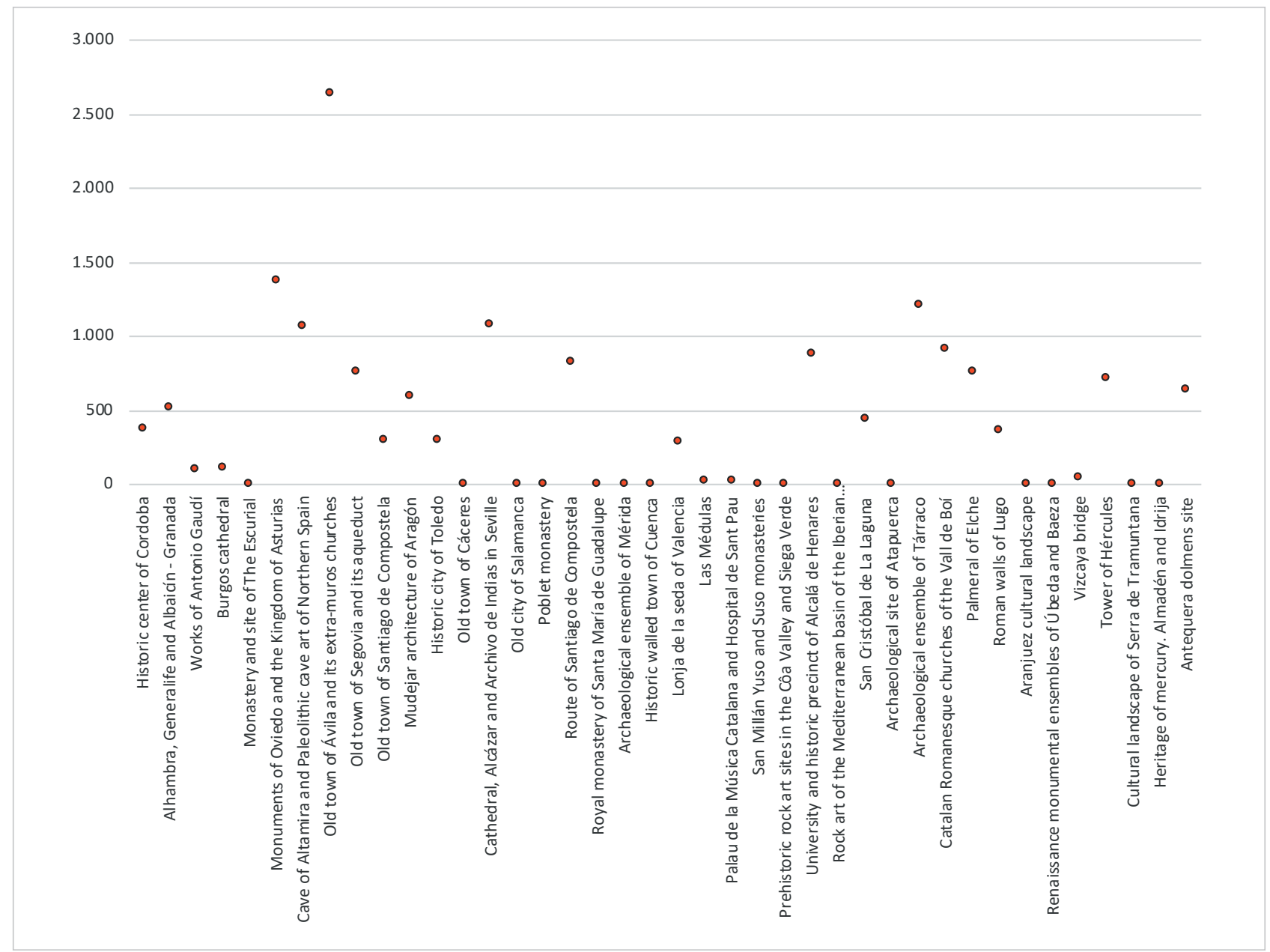

Graph 3. Global engagement on Twitter. The sites are ordered chronologically by the date they were declared World Cultural Heritage, as stated by the Spanish Ministry of Culture and Sport.

Graph 3 shows the global engagement generated on Twitter. The representation of this parameter allows us to compare, on the one hand, the global engagement index generated with WHS through events 2.0 promoted in this platform; and on the other, to detect differences in terms of the engagement generated through the social network Facebook (Graph 1).

There are three WHS that stand for their effective generation of engagement in tourist users:

- Old town of Ávila and its extra-muros Churches (value = 2,641);

- Monuments of Oviedo and the Kingdom of Asturias (Value = 1,373); and

- Archaeological ensemble of Tárraco (value = 1,212).

There is a coincidence in the generation of global engagement through Facebook in the case of the first two WHS.

In contrast, there are nine WHS that do not include events within their marketing strategies:

- Old town of Cáceres;

- Old city of Salamanca;

- Archaeological ensemble of Mérida;

- San Millán Yuso and Suso monasteries;

- Prehistoric rock art sites in the Côa Valley and Siega Verde;

- Rock art of the Mediterranean basin on the Iberian Peninsula;

- Archaeological site of Atapuerca;

- Renaissance monumental ensembles of Úbeda and Baeza; and

- Heritage of mercury: Almadén and Idrija.

Of all these WHS, only the first three have organized various events 2.0 on Facebook.

Graph 4 represents only the positive enga-

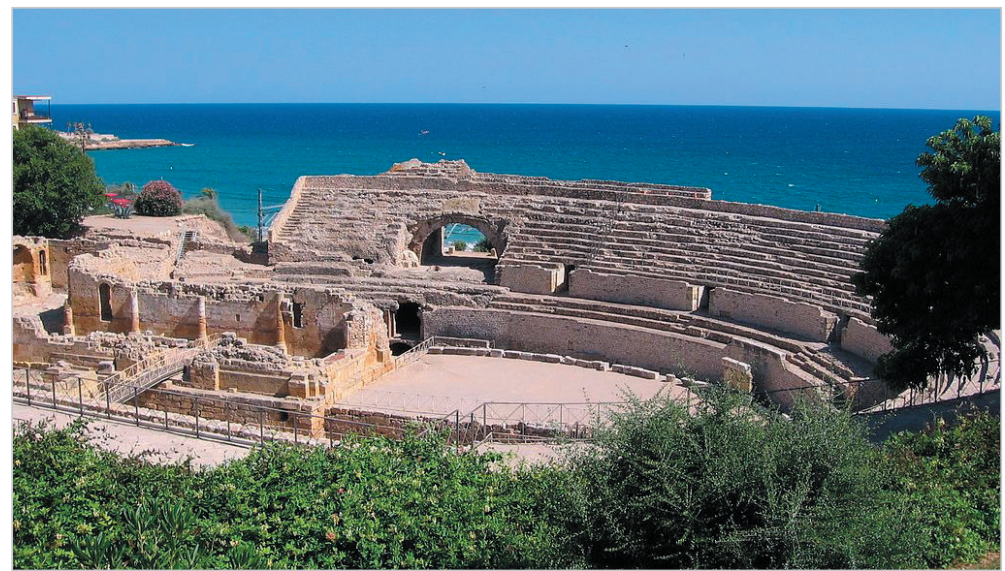

Archaeological Ensemble of Tárraco. Amphitheater.

https://es.wikipedia.org/wiki/Anfiteatro_de_Tarraco 
Graph 4. Positive engagement on Twitter

gement index obtained by the World Heritage Sites on Twitter, and the irrelevance of the negative value as a result of research is again confirmed (the negative comments represent $12.12 \%$ of the total). Two levels of commitment are established accordingly through the median.

WHS were segmented into two groups ( $\mathrm{A}$ and $\mathrm{B}$ ) according to their actions on Twitter to highlight the most effective WHS in the generation of positive engagement:

- Group A contains the aforementioned three WHS that have generated the highest global engagement index (Graph 3) on Twitter;

- Group B contains the WHS with the lowest efficiency rate in the generation of engagement through the promotion of events through this social network.

\section{Conclusions}

The events 2.0 that are integrated into the promotion and commercialization strategies of WHS have become relevant tourist resources that produce synergies with the structural offer made by the destination. They also counteract seasonality in the tourism industry and contribute to a significant increase in the rate of visits and overnight stays.

These events are based on the inbound philosophy, are promoted through integrated user-oriented communication strategies and contribute, along with other

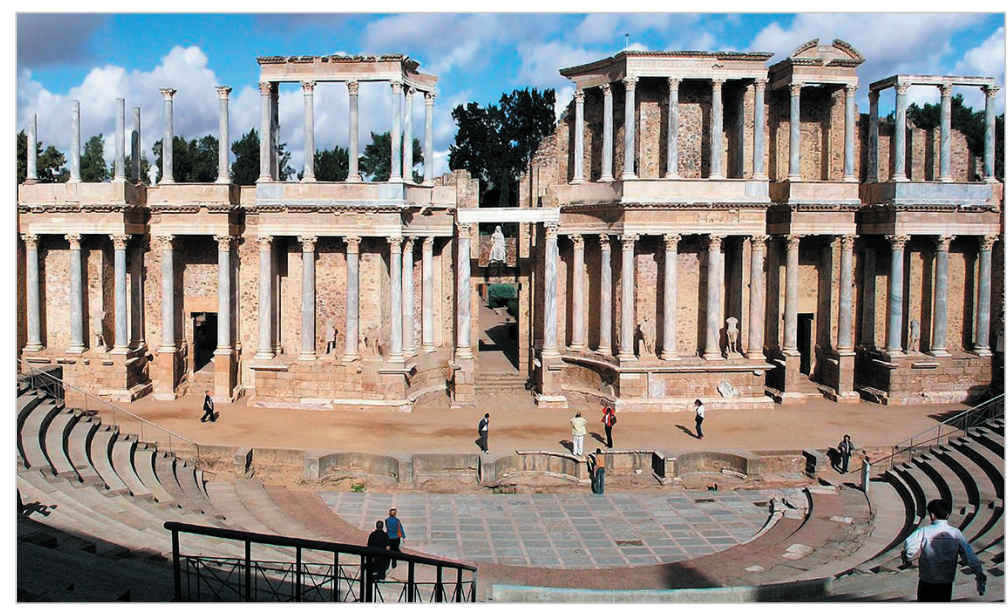

Archaeological site of Mérida. Theater.

https://upload.wikimedia.org/wikipedia/commons/9/93/Collage_de_Mérida.jpg 
actions designed by destination marketing organization (DMO), to the generation of positive emotional connections (engagement) with territorial brands. This process uses transmedia branding to integrate the historical, cultural and artistic values of the WHS brand in a multichannel narrative that provides an integrated experience of the event through social networks.

Online engagement, as a behavioral manifestation of the events, is assessed according to the degree of participation and interaction in social media. The different levels of interaction (reactions, sharing and comments) allow DMO to measure the global engagement (positive and negative) index they have generated. The proactive role of tourist users conditions their activity, since the monitoring and control of the social network accounts of WHS allow the detection of negative opinions and comments that should be handled to avoid the generation of negative engagement towards these destinations. However, the exploratory analysis indicates that this activity is carried out in a very residual way.

The results of this research confirm that there are substantial differences in the management of events 2.0 by the 40 WHS, as reflected in the data on participation and interaction of the tourist user in the social networks Facebook and Twitter.

For WHS marketing organizations, the measurement of engagement in events 2.0 allows to evaluate user's cognitive engagement and emotional connection with the territorial brand. This parameter is a compulsory reference in the design of new events linked to the promotion of WHS.

The planning and management of events 2.0 that are integrated in the marketing strategies of WHS are key elements in the management of their online reputation. Accordingly, their structural development and communication must be carried out with efficiency, profitability and identification of the destination as intrinsic value.

\section{References}

Altamirano, Verónica; Túñez, Miguel (2016). “Promoción y difusión turística de los países de lberoamérica a través de los medios sociales". International journal of information systems and tourism, v. 1, n. 1, pp. 76-90.

http://www.uajournals.com/ojs/index.php/ijist/article/view/122

AIMC (2017). 19o Navegantes en la Red. Encuesta AIMC a usuarios de internet 2016. Asociación para la investigación de medios de comunicación, AIMC.

https://www.aimc.es/a1mc-cOnt3nt/uploads/2017/05/macro2016ppt.pdf

Batthyány, Karina; Cabrera, Mariana (coords.) (2011). Metodología de la investigación en ciencias sociales. Apuntes para un curso inicial. Montevideo: Universidad de la República. ISBN: 9789974007697

http://cienciassociales.edu.uy/departamentodesociologia/wp-content/uploads/sites/3/2013/archivos/FCS_ Batthianny_2011-07-27-imprimir.pdf

Biz, Alexandre-Augusto; Santos, Clara-Kamila; Michelloti-Bettoni, Eduardo; Mendez-Thomas, Guilherme (2016). "Análise do conteúdo veiculado pelas secretarias de turismo das cidades e estados-sedes da copa do mundo 2014 em suas páginas do Facebook". Pasos. Revista de turismo y patrimonio cultural, v. 14, n. 2, pp. 543-559.

http://www.pasosonline.org/Publicados/14216/PS216_16.pdf

Campillo-Alhama, Concepción (2012). “El desarrollo de políticas estratégicas turísticas a través de la marca acontecimiento en el municipio de Elche (2000-2010)". Pasos. Revista de turismo y patrimonio cultural, v. 10, n. 1, pp. 119-129. https://dialnet.unirioja.es/servlet/articulo?codigo=3870762

Campillo-Alhama, Concepción; Martínez-Sala, Alba-María (2017). “Integrated communication 2.0 in municipal administration". El profesional de la información, v. 26, n. 3, pp. 507-515.

https://doi.org/10.3145/epi.2017.may.17

Caro, José-Luis; Luque, Ana; Zayas, Belén (2015). “Nuevas tecnologías para la interpretación y promoción de los recursos turísticos culturales". Pasos. Revista de turismo y patrimonio cultural, v. 13, n. 4, pp. 931-945.

http://www.pasosonline.org/es/articulos/817-nuevas_tecnologas_para_la_interpretacin_y_promocin_de_los_ recursos_tursticos_culturales

Chan-OImsted, Sylvia; Wolter, Lisa-Charlotte (2017). "Toward a multidimensional framework of media engagement: Conceptualizing consumer experience and connection with media content in a digital environment". In: Emma conf 2017. European Media Management Association.

https://www.media-management.eu/ocs/index.php/emma/emma2017/paper/view/585

Domínguez-Vila, Trinidad; Araújo-Vila, Noelia (2012). “El fenómeno 2.0 en el sector turístico. El caso de Madrid 2.0". Pasos. Revista de turismo y patrimonio cultural, v. 10, n. 3, pp. 225-237.

http://www.pasosonline.org/Publicados/10312/PS0312_01.pdf

Ejarque-Bernet, Josep (2016). Marketing y gestión de destinos turísticos: Nuevos modelos y estrategias 2.0. Madrid: Pirámide. ISBN: 9788436835106 
Event Marketing Institute; Freeman XP (2015). The viral impact of events: Extending \& amplifying event reach via social media. Event Marketing Institute; Freeman XP

http://cdn.freemanxp.com/documents/1382/the_viral_impact_of_events_study_freemanxp_and_emi_final.pdf

Fernández-Castrillo, Carolina (2014). "Prácticas transmedia en la era del prosumidor: Hacia una definición del contenido generado por el usuario (CGU)". Cuadernos de información y comunicación, v. 19, pp. 53-67.

https://doi.org/10.5209/rev_CIYC.2014.v19.43903

Fernández-Vazquez, Jessica; López-Rodríguez, Carla; Arévalo-Iglesias, Lorena (2016). "Turismo cultural y nuevas tecnologías de la información: el caso del Camino de Santiago y el fomento de la marca país". Question. Revista especializada en periodismo y comunicación, v. 1, n. 49, pp. 241-251.

http://oaji.net/articles/2016/3111-1459778004.pdf

Folgado-Fernández, José-Antonio (2014). Influencia de la marca de los eventos en la formación de la imagen del destino turístico: una contribución a la explicación de la fidelidad. Tesis doctoral. Universidad de Extremadura.

http://hdl.handle.net/10662/1359

Galmés-Cerezo, María-Asunción (2010). La organización de eventos como herramienta de comunicación de marketing. Modelo integrado y experiencial. Tesis doctoral. Universidad de Málaga. ISBN: 9788497476096 https://libros.metabiblioteca.org/bitstream/001/356/5/978-84-9747-609-6.pdf

Gómez-Oliva, Andrea; Server-Gómez, Matilde; Jara, Antonio J.; Parra-Meroño, María-Concepción (2017). “Turismo inteligente y patrimonio cultural: un sector a explorar en el desarrollo de las smart cities". International journal of scientific management and tourism, v. 3, n. 1, pp. 389-411.

https://dialnet.unirioja.es/servlet/articulo?codigo $=5975077$

González-Teruel, Aurora (2015). “Estrategias metodológicas para la investigación del usuario en los medios sociales: análisis de contenido, teoría fundamentada y análisis del discurso”. El profesional de la información, v. 24, n. 3, pp. 321328.

https://doi.org/10.3145/epi.2015.may.12

Grupo Ciudades Patrimonio de la Humanidad de España (2018).

http://www.ciudadespatrimonio.org/mpublicaciones/patrimonio.php

Harrigan, Paul; Evers, Uwana; Miles; Morgan; Daly, Timothy (2017). “Customer engagement with tourism social media brands". Tourism management, v. 59, pp. 597-609.

https://doi.org/10.1016/j.tourman.2016.09.015

Hays, Stephanie; Page, Stephen-John; Buhalis, Dimitrios (2013). "Social media as a destination marketing tool: its use by national tourism organisations". Current issues in tourism, v. 16, n. 3, pp. 211-239.

https://doi.org/10.1080/13683500.2012.662215

Huertas-Roig, Assumpció (2014). La comunicación de los territorios, los destinos y sus marcas. Barcelona: UOC. ISBN: 9788490642078

Huertas-Roig, Assumpció; Setó-Pàmies, Dolors; Míguez-González, María-Isabel (2015). “Comunicación de destinos turísticos a través de los medios sociales". El profesional de la información, v. 24, n. 1, pp. 15-21.

https://doi.org/10.3145/epi.2015.ene.02

IAB Spain (2017). Estudio anual de redes sociales 2017. Interactive Advertising Bureau, IAB Spain. https://iabspain.es/wp-content/uploads/iab_estudioredessociales_2017_vreducida.pdf

Jiménez-Morales, Mónika; De-San-Eugenio-Vela, Jordi (2009). “Identidad territorial y promoción turística: la organización de eventos como estrategia de creación, consolidación y difusión de la imagen de marca del territorio". ZER. Revista de estudios de comunicación, v. 14, n. 26, pp. 277-297.

https://www.ehu.eus/ojs/index.php/Zer/article/view/2776?source=/ojs/index.php/Zer/article/view/2776

Lara-de-Vicente, Fernando; López-Guzmán-Guzmán, Tomás J. (2004). “El turismo como motor de desarrollo económico en ciudades patrimonio de la humanidad". Pasos. Revista de turismo y patrimonio cultural, v. 2, n. 2, pp. $243-256$.

http://mail.pasosonline.org/Publicados/2204/PS070204.pdf

Lara-Navarra, Pablo; López-Borrull, Alexandre; Sánchez-Navarro, Jordi; Yànez, Pau (2018). “Medición de la influencia de usuarios en redes sociales: propuesta SocialEngagement". El profesional de la información, v. 27, n. 4, pp. 899-908. https://doi.org/10.3145/epi.2018.jul.18

Lenderman, Max (2008). Marketing experiencial. La revolución de las marcas. Madrid: Editorial ESIC. ISBN: 978847356 5455 
Mariani, Marcello M.; Di-Felice, Marco; Mura, Matteo (2016). "Facebook as a destination marketing tool: Evidence from Italian regional destination management organizations". Tourism management, v. 54, pp. 321-343.

https://doi.org/10.1016/j.tourman.2015.12.008

Mariné-Roig, Estela (2017). "Measuring destination image through travel reviews in search engines". Sustainability, v. 9, n. 8, p. 1425.

https://doi.org/10.3390/su9081425

Mariné-Roig, Estela; Huertas-Roig, Assumpció (2016). “Metodologías de análisis”. En: Huertas-Roig, Assumpció (ed.). La comunicación de los destinos turísticos y sus marcas a través de los medios sociales, pp. 9-16. Tarragona: Universitat Rovira i Virgil.

https://www.segittur.es/opencms/export/sites/segitur/.content/galerias/descargas/documentos/libro-COMTUR_2.0.pdf

Martínez-Sala, Alba-María (2018). "Marketing 2.0 aplicado al sector turístico: la función comercial de los sitios webs de las organizaciones de marketing de destinos". Vivat academia, n. 143, pp. 1-23.

https://doi.org/10.15178/va.2018.143.01-23

Martínez-Sala, Alba-María; Monserrat-Gauchi, Juan; Campillo-Alhama, Concepción (2017). "El paradigma relacional en las estrategias de las organizaciones de marketing de destinos. Interactividad y función social en las webs turísticas oficiales". Revista latina de comunicación social, n. 72, pp. 374-396.

https://doi.org/10.4185/RLCS-2017-1170

Martos-Molina, Marta (2013). "El papel del turismo de eventos en el desarrollo urbano. El caso de Expo Zaragoza”. Pasos. Revista de turismo y patrimonio cultural, v. 11, n. 1, pp. 57-71.

http://www.pasosonline.org/Publicados/11113/PS0113_05.pdf

Mendes-Thomaz, Ghilerme; Biz, Alexandre-Augusto; Gândara, José-Manoel (2013). “Innovación en la promoción turística en medios y redes sociales: un estudio comparativo entre los destinos". Estudios y perspectivas en turismo, v. 22, n. 1, pp. 102-119.

https://dialnet.unirioja.es/servlet/articulo?codigo $=5131415$

Pérez-Vega, Rodrigo; Taheri, Babak; Farrington, Thomas; O’Gorman, Kevin (2018). “On being attractive, social and visually appealing in social media: The effects of anthropomorphic tourism brands on Facebook fan pages". Tourism management, v. 66, pp. 339-347.

https://doi.org/10.1016/j.tourman.2017.11.013

Pompurová, Kristina; Marčeková, Radka; Šebová, Ĺubica; Sokolová, Jana; Žofaj, Matej (2018). “Volunteer tourism as a sustainable form of tourism. The case of organized events". Sustainability. v. 10, n. 5, p. 1468.

https://doi.org/10.3390/su10051468

Tenderich, Burghardt (2014). Transmedia branding. Berlin, Germany: Eimo. ISBN: 9781925128208

Troitiño-Vinuesa, Miguel-Ángel (2000). "Turismo y ciudades históricas: retos y oportunidades". Turismo y patrimonio, n. 1, pp. 27-34.

http://ojs.revistaturismoypatrimonio.com/index.php/typ/article/view/42

Turespaña (2017). Plan del turismo español Horizonte 2020.

https://www.tourspain.es/es-es/Conozcanos/Documents/HistoricoPoliticaTuristica/PlanTurismoEspanolHorizonte2020.pdf

Unesco (2018). Lista del patrimonio mundial.

https://whc.unesco.org/es/list

Unwto (2016). Unwto annual report 2015. Madrid: Unwto. ISBN: 9789284418022

http://cf.cdn.unwto.org/sites/all/files/pdf/annual_report_2015_Ir.pdf

Xiang, Zheng; Gretzel, Ulrique (2010). "Role of social media in online travel information search". Tourism management, v. 31 , pp. $179-188$.

https://doi.org/10.1016/j.tourman.2009.02.016 